\title{
BMJ Open Determinants of patient delay in doctor consultation in head and neck cancers (Protocol DEREDIA)
}

\author{
Véronique Christophe, ${ }^{1,2,3}$ Tanguy Leroy, ${ }^{1,4}$ Mélanie Seillier, ${ }^{1,5}$ Camille Duthilleul, ${ }^{1}$ \\ Morbize Julieron, ${ }^{6}$ Stéphanie Clisant, ${ }^{6}$ Jérôme Foncel, ${ }^{7}$ Fanny Vallet, ${ }^{1}$ \\ Jean-Louis Lefebvre ${ }^{6}$
}

To cite: Christophe V, Leroy T, Seillier M, et al. Determinants of patient delay in doctor consultation in head and neck cancers (Protocol DEREDIA). BMJ Open 2014;4:e005286. doi:10.1136/bmjopen-2014005286

- Prepublication history for this paper is available online. To view these files please visit the journal online (http://dx.doi.org/10.1136/ bmjopen-2014-005286)

Received 18 March 2014 Revised 3 July 2014 Accepted 7 July 2014

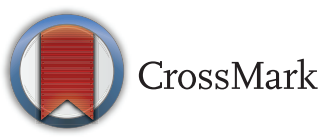

For numbered affiliations see end of article.

Correspondence to Véronique Christophe; veronique.christophe@univlille3.fr

\section{ABSTRACT}

Introduction: Reducing the time between the onset of the first symptoms of cancer and the first consultation with a doctor (patient delay) is essential to improve the vital prognosis and quality of life of patients. Longer patient delay is linked to the already known sociodemographic, socioeconomic, socioeducational, sociocultural and socioprofessional factors. However, recent data suggest that some sociocognitive and emotional determinants may explain patient delay from a complementary point of view. The main objective of this study is to assess whether, in head and neck cancer, patient delay is linked to these sociocognitive and emotional factors, in addition to previously known factors.

Methods and analysis: We intend to include in this study 400 patients with a not yet treated head and neck cancer diagnosed in one of six health centres in the North of France region. The main evaluation criterion is 'patient delay'. Sociocognitive, emotional, medical, sociodemographic, socioeconomic, educational, professional and geographic factors will be assessed by means of (1) a case report form, (2) a questionnaire completed by the clinical research associate together with the patient, (3) a questionnaire completed by the patient and (4) a recorded semidirective interview of the patient by a psychologist (for 80 patients only). The collected data will be analysed to underline the differences between patients who consulted a doctor earlier versus those who consulted later.

Ethics: The study has obtained all the relevant authorisations for the protection of patients enrolled in clinical trials (CCTIRS, CCP, CNIL), does not involve products mentioned in article L.5311-1 of the French Code of Public Health, and does not imply any changes in the medical care received by the patients. The study began in October 2012 and will end in June 2015.

Trial registration: ID-RCB 2012-A00005-38.

\section{BACKGROUND}

The time between the onset of the first symptoms and the effective treatment of patients with cancer seems to be a decisive factor in the vital prognosis of patients ${ }^{12}$ and in the psychological adjustment of patients and their relatives. ${ }^{3}$ In fact, the different intervals composing this delay (patient, primary care and secondary care intervals) have direct consequences on the tumour stage at diagnosis and on short-term survival. ${ }^{4}$ Therefore, reducing mortality related to avoidable cancers implies reducing the timeline between the initial diagnosis of the disease and the beginning of medical treatment. In addition to the time required for the prescription of medical examinations, the carrying out of examinations, the diagnosis of cancer and treatment initiation, the delay between the onset of the first symptoms and the patient's consultation with a doctor seems to be a decisive factor in survival and

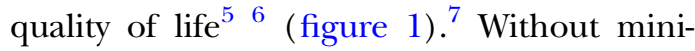
mising the time attributable to the structure of the healthcare system as such, it appears essential to reduce the time between the onset of the first disease-related symptoms and the first mention of these symptoms by the patient to a doctor (patient delay).

The question of patient delay has been extensively studied over the past few years. A study conducted in Denmark shows that for all cancers combined, the median delay between the onset of the first symptoms and the consultation for medical advice is 3 weeks. ${ }^{6}$ Based on studies carried out in Scotland and England, ${ }^{8} 9$ the median delay for patients with head and neck cancers is around 30 days, which appears to be longer than for other tumour locations. More precisely, patients with head and neck cancer show the longest delay among 13 cancers $^{8}$ and patients with oropharyngeal cancer show the longest delay among 18 cancers. $^{9}$ This longer delay among these patients can be partially explained by the impact of 


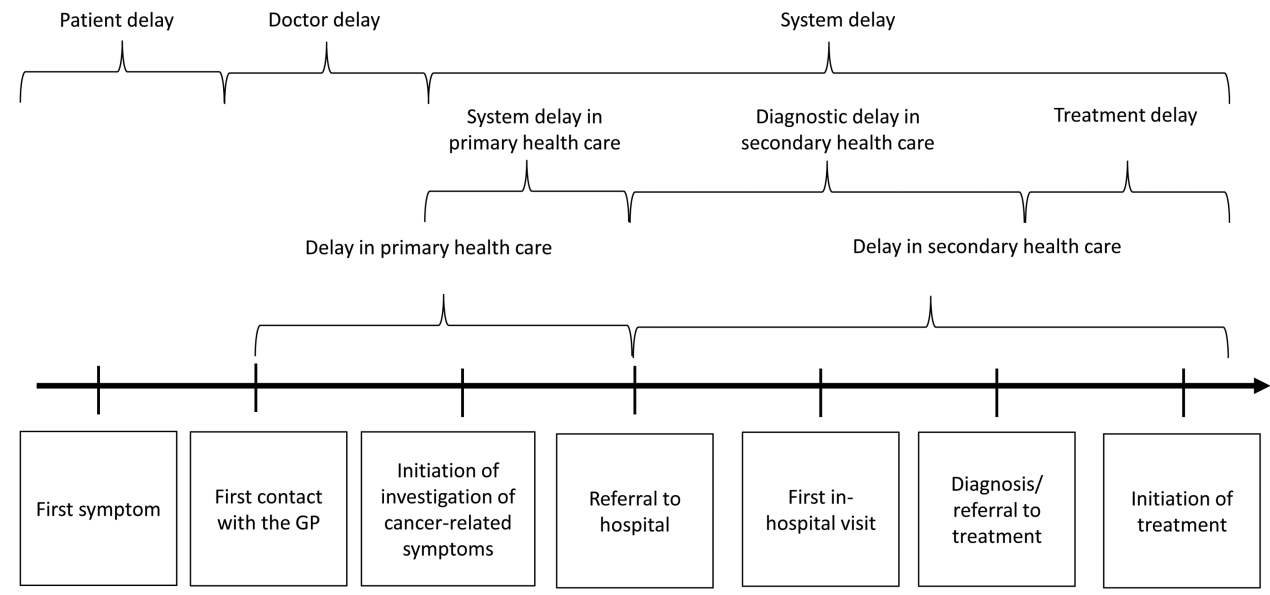

Figure 1 Different types of delay between the onset of the first symptoms and the beginning of anticancer treatment. ${ }^{7}$

sociodemographic, socioeconomic, socioeducational and sociocultural factors such as gender, age, socioprofessional category or the level of income or education. ${ }^{3}{ }^{10-}$ ${ }^{13}$ Other studies also emphasise the influence of psychosocial and behavioural differences with regard to smoking and alcohol consumption. ${ }^{14}$ Nevertheless, these well-known factors do not seem to explain entirely the delay before consulting for these types of cancer; further clarification may be provided by a number of sociocognitive and emotional factors.

In some diseases, for example, it has been shown that delayed consultation may be related to the patients becoming aware of their symptoms, how they assess these symptoms, their emotional impact ${ }^{15-17}$ and the coping strategies implemented to deal with them. ${ }^{10} 1819$ The participant's social and family environment and the social support received also appear to be decisive factors. ${ }^{20-22}$ These components have been underlined by the model of Pathways to Treatment and empirical data, ${ }^{23}{ }^{24}$ which distinguish between the appraisal interval ("the time from the detection of a bodily change to perceiving a reason to discuss symptoms with a HCP (healthcare provider)' and the help-seeking interval ('the time from perceiving a reason to discuss symptoms with a HCP to the first consultation with a HCP about their symptoms'). ${ }^{23}$ The main determinants identified in the literature cover these different steps from the perception and interpretation of the symptoms (eg, gravity), to emotional regulation and coping (eg, avoidance), then to the perception of a reason to consult a doctor and the decision to consult him/her actually (eg, perceived costs and benefits, self-efficacy and outcome expectations). ${ }^{23}$ More generally, based on the results of explanatory models used to analyse the factors influencing health behaviour, ${ }^{25-30}$ the decision to consult a doctor after the onset of the first symptoms appears to be determined by the following:

- Subjective perceptions concerning health: the patient's beliefs and perception of cancer, ${ }^{31}{ }^{32}$ feelings of vulnerability in relation to the disease, assessment of the gravity of the perceived symptoms, ${ }^{25}$ as well as reappraisal of some warning signs of cancer, ${ }^{33}$ which is particularly relevant for head and neck cancers, as some symptoms are common and may be misattributed ${ }^{23}$

- Perception of medical care the patient's perception of treatments and, more specifically, benefits or, to the contrary, fatalism regarding cancer ${ }^{34}$; perceived barriers to the seeking of medical advice ${ }^{35-37}$ especially how patients perceive their capacity to explain their symptoms to a doctor and consequently to take part in a treatment protocol and submit themselves to medical prescriptions ${ }^{26}{ }^{27}$;

- Social incitation to consult a doctor: from the patient's relatives and the healthcare system; the patient's acceptance of being influenced by the incitation of others ${ }^{25} 28$;

- Emotional factors: the emotions produced by the onset of the symptoms, emotion regulation strategies implemented to cope with the situation, difficulties experienced in doing so and any potential social support received $^{29}$;

- Structural or environmental constraints: the subjective priority granted by the patient to resolve situational difficulties caused by changes in living conditions (loss of revenue, vulnerable family structure, financial insecurity, geographic distance from the healthcare centre, etc). ${ }^{27} 38$

This study aims to identify the factors explaining delayed consultation for head and neck cancers in the North of France (Nord - Pas de Calais region), where such cancers are particularly prevalent. Understanding which factors mostly determine the behaviour of consulting a doctor when the first cancer symptoms appear seems to be essential for the adjustment and optimisation of preventive messages in public health. In this context, the use of theoretical health decision models seems particularly suitable to approach this question of consultation delay from a global perspective. In order to modify patient health behaviour, thus improving their 
vital prognosis and quality of life as well as reducing social inequalities regarding health, it seems essential to take into account not only patient representations concerning health but also the social, emotional and contextual determinants of their decisions and behaviour.

This study aims to reveal the sociocognitive and emotional factors associated with delayed consultation of a doctor following the onset of the first symptoms of cancer (patient delay). More precisely, the goal is to determine whether patient delay is related to:(1) subjective perceptions concerning health, the disease, means of treatment, the healthcare system, the patient's capacity for action and self-efficiency, (2) emotion regulation strategies and difficulties, (3) the perceptions and behaviour of the patient's relatives with regard to healthcare and prevention, (4) the information received and its sources and (5) other situational difficulties.

The secondary objectives of the study are: (1) to identify which medical, sociodemographic, socioeconomic, socioprofessional, socioeducational and geographic variables are related to delayed consultation of a doctor following the onset of the first symptoms and (2) to ascertain the sources (attributable to the patient, the doctor or the healthcare system) that most delay the final diagnosis of the disease and to determine which variables among those cited above are associated with these sources of delay based on how the patients were managed medically between the onset of the first symptoms and diagnosis.

\section{METHODS/DESIGN}

The study has obtained all the relevant authorisations for the protection of patients enrolled in clinical trials (CCTIRS, CCP, CNIL), does not involve products mentioned in article L.5311-1 of the French Code of Public Health and does not imply any changes in the medical care received by the patients. The study began in October 2012 and will end in June 2015.

\section{Population}

To be included in the study, patients must be over 18 years of age and have a cancer of the oral cavity (tongue, floor of the mouth, gums, palate and inside of the cheeks), of the oropharynx (tonsils, root of the tongue and soft palate) or of the hypopharynx and larynx (supraglottis, glottis and subglottis). Only untreated patients will be invited to participate in the study, before any cognitive reappraisal of the situation induced by surgery, radiation therapy or medicinal treatment. Patients must be aware of their cancer diagnosis, understand and speak French fluently and sign an informed consent to participate in the study. Exclusion criteria are cancer antecedents or psychiatric disorders liable to alter the patient's reasoning, discerning or judgemental abilities.

The 'interview' subgroup will comprise patients who comply with all the aforementioned selection criteria, who present no speech impediments and who agree to their comments being recorded.

\section{Number of patients and sample representativeness}

The representativeness of our sample on a national scale will be ensured by patient recruitment in the main institutions responsible for treating head and neck cancers in the North of France (Nord-Pas-de-Calais region) where the incidence of this disease is particularly high. Patients treated in these centres come from the four large areas of the region with very different sociocultural histories, which implies considerable sociodemographic and socioprofessional diversity: the Lille metropolis (Oscar Lambret Center, Lille Regional University Hospital Centre, La Louvière private hospital), the coastal area (Coastal Specialised Medical Centre, Boulogne-sur-Mer Hospital Centre), the mining area (Lens Hospital Center) and Avesnois (patients mostly taken care of in the Lille metropolis). Sample representativeness will also be ensured by the participation of both state-run and private hospitals.

Given the nature of the investigation, it is not possible to calculate the number of participants required for the study. This will therefore be determined according to the feasibility of the study. The annual incidence of head and neck cancers in the Nord-Pas-de-Calais region is 4000 . This multicentre study will be performed in several hospitals which manage approximately $90 \%$ of patients with such cancers in the region. The sample will be composed of 400 patients in total, comprising 200 with early consultation and 200 with delayed consultation.

For the 'interview' subsample, the answers given to an open question during semidirective interviews often become redundant after around 20 participants. In order to enable a statistical comparison of the two groups of patients (early vs delayed consultation) and in view of the fact that the patients can only be separated into the two groups after all the data have been collected, the subsample has been enlarged to include 80 participants per group. This will enable statistical inference while preserving the feasibility of the study in terms of data analysis, since qualitative analysis of semidirective interviews is particularly time consuming.

\section{Assessment criteria}

Main assessment criterion

The main assessment criterion is the time, in weeks, between the onset of the first cancer-related symptoms and the first time the patient makes an appointment to talk to a doctor about these symptoms. This patient delay will be estimated based on three different reports to optimise data validity:

1. what the patient reports to the investigator (patient delay reported by the investigator),

2. what the patient reports to the clinical research associate during completion of the face-to-face questionnaire (patient delay reported by the clinical research associate), 
3. what the doctor who first saw the patient reports: the general practitioner or specialist will be contacted by phone after obtaining the patient's agreement ( patient delay reported by the general practitioner or private practice specialist).

\section{Secondary assessment criteria}

The secondary assessment criteria concern the patient's medical data and sociodemographic, socioeconomic, socioeducational and geographic indicators, as well as sociocognitive and emotional indicators. The assessments will be made by: (1) completion of a case report form (CRF), (2) completion of a face-to-face questionnaire by a clinical research associate with the patient, (3) completion of a self-assessment questionnaire by the patient and (4) the carrying out of a semidirective interview with the patient by a competent psychologist mandated by the sponsor:

1. CRF: primary location of the cancer, TNM classification at the stage of initial diagnosis, history of main medical and surgical events, current symptoms and treatments, treatment dates and modalities since the patient entered the treatment process;

2. Face-to-face questionnaire: sociodemographic, socioprofessional, socioeconomic and socioeducational indicators (gender, age, place of residence, lifestyle, professional activity, annual revenue, last diploma obtained, family history of chronic diseases), usual health behaviours (frequency of consultations, tobacco and alcohol consumption), current symptoms (presence or not, duration and perceived link with cancer from the patient's point of view) and date of the onset;

3. Self-assessment questionnaire. sociocognitive and emotional determinants of the medical appointment that resulted in the detection of cancer. Based on theoretical models, items-seven-point Likert scales-have been specifically built with a view to understanding delay determinants:

- Subjective perceptions concerning health: importance given to health and perceived vulnerability to diseases before the onset of the first symptoms (3 items); first symptoms of the disease: perceived gravity and anxiety in relation to the symptoms ( 2 items);

- Perception of medical care: feeling of control regarding a possible treatment: perceived costs, perceived benefits and self-efficacy ( 5 items);

- Social incitation to consult: incitation from relatives and value given to this incitation (2 items); sources of medical information: relatives, media, pharmacist (3 items);

- Emotional factors: emotional state: anxiety and depression moods (4 items); emotion regulation difficulties and strategies: difficulty in cooling down-emotion regulation strategies (avoidance, reappraisal, social sharing of emotions, symptomcentered coping) (5 items);
- Structural or environmental constraints: health may not be a priority; patient faced with other practical difficulties (2 items).

4. Semidirective interview only for patients in the interview' subsample: determinants of the medical appointment that resulted in the patient taking part in an anti-cancer treatment protocol, specifically, on the one hand, subjective perceptions concerning the symptoms and the medical appointment and, on the other hand, subjective perceptions of health, the disease and treatments.

\section{Study conduct}

All the patients complying with all the selection criteria will be included in the study. Eligibility forms will be filled in by the investigator to ensure that the patient complies with all the selection criteria. The investigator will suggest the study to the patient and, if the latter agrees, the investigator will give the patient the information letter and ask them to sign the informed consent form. The patient will then be given an identification number corresponding to their chronological order of inclusion in the investigator's centre. The eligibility form with the patient's identification number will be sent to the study sponsor so that their inclusion may be recorded.

The clinical research associate will be in charge of filling in the CRF for the study. For patients who are not in the 'interview' subsample, an appointment will be made with a clinical research associate. He/she will fill in the face-to-face questionnaire with the patient. After that, the patient will fill in the self-assessment questionnaire. With the patient's agreement, the sponsor might recontact them or their general practitioner at a later date to obtain any data that might be missing from the CRF or the face-to-face questionnaire.

For patients in the 'interview' subsample, a first appointment will be made with a psychologist who will conduct the semidirective interview which will be recorded (using a digital recorder) in compliance with the standardised procedure. Then the patient will meet the clinical research associate for the face-to-face questionnaire and the self-assessment questionnaire.

In order not to constrain patients, appointments will always be planned for when patients are hospitalised (generally for complementary examinations), between the cancer diagnosis announcement and the beginning of treatment. The visit will take place in the patient's hospital room.

\section{Analyses}

Once the patient selection criteria have been checked, the statistical analyses will be performed by the URECA EA 1059 and EQUIPPE EA 4018 Research Units of Lille 3 University.

A. Quantitative analyses

The geographic, sociodemographic, socioprofessional, socioeconomic, socioeducational, sociocognitive, emotional and medical data will be presented in 
recapitulative or contingency tables summarising the typical parameters used in descriptive statistics (frequencies, percentages, means, SDs depending on whether the variables are categorical or continuous). Contingency tables may be established for the different variables and targets if justified. Intergroup comparisons will be performed on these quantitative data using common parametric inferential statistics (ANOVA, MANOVA, Student's t test) and non-parametric statistics $\left(\chi^{2}\right.$, rank tests) to determine the differences, based on the different variables, between patients who consulted a doctor early versus those who consulted at a later stage after the onset of the first symptoms. Early versus late consultation groups will be defined on the basis of the median of the averages of the three measures of delay (patient delay reported by the investigator, by the clinical research associate, and by the general practitioner or private practice specialist).

Ultimately, duration models (including the Cox model $^{39}$ ) will be used to identify the causal and explanatory factors for delayed consultation. The results of the estimates will then be used for the implementation of preventive actions aimed at reducing delays. From a technical perspective, in order to obtain robust results, it will also be possible to check for potential assessment errors in the recording of consultation delays. Error measurement can be interesting for retrospective data collection based on the patients remembering events that might have occurred a long time beforehand. Tests are available to determine whether the assessment error is significant or not ${ }^{40}$ and customised models may be applied to take into account any such potential assessment errors. ${ }^{41}$

B. Qualitative analysis

The semidirective interviews of the patients from the 'interview' subsample will be analysed qualitatively to identify the factors leading to the first medical consultation that resulted in the patient taking part in a treatment protocol. This step will be applied to all the interviews, regardless of the time taken by patients to consult their doctor. When all the determinants of the medical consultation have been identified, the data will be synthesised by grouping the factors into categories. Descriptive and inferential quantitative analyses will then be performed for each of the two groups of patients (early vs late consultation) in order to compare how frequently the various factors were mentioned in the two groups to determine the factors associated with early consultation versus those associated with late consultation. Correlations between these data and the quantitative assessments obtained from the patients will be examined to show the links between the various determinants and medical consultation.

\section{DISCUSSION}

\section{Expected outcomes}

The data collected in this study will be used to evaluate the medical, geographic, sociodemographic, socioprofessional, socioeconomic, socioeducational, sociocognitive and emotional factors affecting the consultation timeline after the onset of the first symptoms of head and neck cancers. This will help reveal the factors responsible for late diagnosis of patients suffering from these cancers. Given that the study considers medical, social, cognitive and emotional factors, it will confirm or invalidate the observations reported in the literature. Moreover, the results about psychosocial determinants will be analysed and discussed to gain a broader theoretical understanding of the processes of deciding to consult (appraisal and help-seeking inter$\mathrm{val}^{23}$ ), in order to identify the appropriate level to offer intervention and/or further investigations. Furthermore, it will enable the issue of late diagnosis to be addressed, taking into account the full variety of factors affecting health behaviour as they have been considered, often separately, in various theoretical models assessing health psychology. Based on these new observations, an index will be established assembling the most discriminatory variables affecting populations at risk of consulting a doctor belatedly after the onset of the first cancer-related symptoms. Fundamentally, the data collected in the study will enable the testing and adjustment of health decision models. The understanding of the factors affecting health-related behaviour will be improved, as will the methods used to prevent pathogenic behaviour and promote protective behaviour.

\section{Strengths and limitations of the design}

Owing to the fact that this design strives to understand the many determinants of delay and has important implications for the literature as well as for implementing interventions, it is important to summarise its strengths and point out its limitations, inspired in part by the Aarhus checklist. ${ }^{42}$ First, the beginning and the end points of the consultation delay are clearly defined and their assessment is therefore replicable. As the recall of the onset of symptoms may involve some recall biases, three measurements with different methods will be used to minimise the measurement error. Moreover, the selfassessment questionnaire including sociocognitive and emotional determinants has been built to assess the specific hypotheses of this study. We have made this choice because (1) given the large range of variables studied, the use of specific validated questionnaires assessing each variable would be impossible, (2) some questionnaires (eg, the importance of health in patients' personal priorities, their perceptions and reactions when they are faced with the onset of the first symptoms) do not exist, or have not yet been validated or are difficult to find and (3) we have taken many precautions to build the scales on the basis of relevant and up-to-date theoretical models, inspired from previous studies, and the relevance and wording of items have been discussed carefully with several researchers involved in the care of head and neck patients with cancer. We are convinced that all these methodological precautions will ensure the reliability of the data collected in this study. 


\section{Potential impacts}

During this study, the identification of new determinants explaining delayed consultation will enable a better targeting of the populations at risk of entering a treatment protocol at a late stage. The results of this study will improve the determination of individual and group factors that may explain patient delay in consulting for medical advice, an essential parameter in the prevention of abnormally high death rates and social inequality with regard to access to healthcare for patients with head and neck cancers. Knowledge of the socioeconomic environment of the patient's living place, combined with individual socioeconomic information, will enable the influence of the context on patient delay to be analysed.

However, it seems premature to design interventional studies straightaway, before the factors that are crucial and necessary for the formalisation and setting up of such studies have been identified. The interests of specific health education systems, personalised screening and healthcare interventions targeting populations at risk of delayed screening are undeniable. Depending on the results of this study, the populations considered at risk may, for example, be offered personalised psychosocial support, access to useful information to help them take health-related decisions, or easier access to the healthcare system and health-promoting systems. In addition, means may be implemented to increase the perceived capacity for action of at-risk populations and their feelings of self-efficiency in terms of health.

The purpose of such operations would be to reduce diagnosis timelines in patients presenting with symptoms suggestive of head and neck cancers, with a view to (1) reducing the abnormally high death rates and changes in quality of life induced by delayed treatment and (2) fighting social inequality in terms of healthcare, a central component of the French Cancer Plan.

\section{Author affiliations \\ ${ }^{1}$ Lille 3 University-URECA EA1059, Villeneuve d'Ascq cedex, France 2U1086 INSERM "Cancers \& Préventions", Université de Caen Basse- Normandie, Avenue de la côte de Nacre, Caen Cedex 5, France \\ ${ }^{3}$ SIRIC ONCOLille-Maison Régionale de la Recherche Clinique, Lille Cedex, France \\ ${ }^{4}$ Aix-Marseille University—Public Health and Chronic Diseases, 3 SIRIC ONCOLille, EA 3279, Marseille cedex 05, France \\ ${ }^{5}$ CERFEP, CARSAT Nord Pas de Calais Picardie, Villeneuve d'Ascq cedex, France \\ ${ }^{6}$ Centre Oscar Lambret-Cervicofacial Oncology Department, Lille cedex, France \\ ${ }^{7}$ Lille 3 University-EQUIPPE EA 4018, Villeneuve d'Ascq cedex, France}

Acknowledgements The authors thank the SIRIC ONCOLille, Grant INCa-DGOS-Inserm 6041, for their help.

Contributors VC, TL, MS, JF and J-LL contributed to the development and design of the protocol. CD and FV drafted the manuscript with critical input from all other authors who have read and approved the final manuscript.

Funding This research was supported by a grant from the Conseil Regional Nord Pas de Calais.

Competing interests None.

Patient consent Obtained.
Ethics approval Clinical research Unit.

Provenance and peer review Not commissioned; externally peer reviewed.

Open Access This is an Open Access article distributed in accordance with the Creative Commons Attribution Non Commercial (CC BY-NC 3.0) license, which permits others to distribute, remix, adapt, build upon this work noncommercially, and license their derivative works on different terms, provided the original work is properly cited and the use is non-commercial. See: http:// creativecommons.org/licenses/by-nc/3.0/

\section{REFERENCES}

1. Jensen AR, Nellemann HM, Overgaard J. Tumor progression in waiting time for radiotherapy in head and neck cancer. Radiother Oncol 2007;84:5-10.

2. Richards MA, Westcombe AM, Love SB, et al. Influence of delay on survival in patients with breast cancer: a systematic review. Lancet 1999;353:1119-26.

3. Risberg T, Sorbye SW, Norum J, et al. Diagnostic delay causes more psychological distress in female than in male cancer patients. Anticancer Res 1996;16:995-9.

4. Lyratzopoulos G. Markers and measures of timeliness of cancer diagnosis after symptom onset: a conceptual framework and its implications. Cancer Epidemiol 2014;38:211-13.

5. Bjerager M. Delay in diagnosis and treatment of lung cancer. [PhD thesis]. 1st edn. Aarhus: Research Unit and Department of General Practice, Faculty of Health Sciences, University of Aarhus, 2006.

6. Hansen RP, Vedsted P, Sokolowski I, et al. Time intervals from first symptom to treatment of cancer: a cohort study of 2,212 newly diagnosed cancer patients. BMC Health Serv Res 2011;11:284.

7. Olesen F, Hansen RP, Vedsted P. Delay in diagnosis: the experience in Denmark. Br J Cancer 2009;101:S5-8.

8. Baughan P, O'Neil B, Fletcher E. Auditing the diagnosis of cancer in primary care: the experience in Scotland. BMJ 2009;101(Suppl 2): S87-91.

9. Keeble S, Abel GA, Saunders CL, et al. Variation in promptness of presentation among 10,297 patients subsequently diagnosed with one of 18 cancers: evidence from a National Audit of Cancer Diagnosis in Primary Care. Int J Cancer 2014;135:1220-8.

10. Hansen RP, Olesen F, Sørensen HT, et al. Socioeconomic patient characteristics predict delay in cancer diagnosis: a Danish cohort study. BMC Health Serv Res 2008;8:49.

11. Hansen RP, Vedsted P, Sokolowski I, et al. General practitioner characteristics and delay in cancer diagnosis: a population-based cohort study. BMC Fam Pract 2011;12:100.

12. Ramirez AJ, Westcombe AM, Burgess CC, et al. Factors predicting delayed presentation of symptomatic breast cancer: a systematic review. Lancet 1999;353:1127-31.

13. Neal RD, Allgar VL. Sociodemographic factors and delays in the diagnosis of six cancers: analysis of data from the 'National Survey of NHS Patients: Cancer'. Br J Cancer 2005;92:1971-5.

14. Brouha X, Tromp D, Hordijk GJ, et al. Role of alcohol and smoking in diagnostic delay of head and neck cancer patients. Acta Otolaryngol 2005;125:552-6.

15. Holtedahl KA. Diagnosis of cancer in general practice. A study of delay problems and warning signals of cancer, with implications for public cancer information and for cancer diagnostic strategies in general practice. ISM skriftserie nr. 16, Universitetet i Tromsø, Institutt for Samfunnsmedisin; 1990.

16. Andersen BL, Cacioppo JT. Delay in seeking a cancer diagnosis: delay stages and psychophysiological comparison processes. $\mathrm{Br} \mathrm{J}$ Soc Psychol 1995;34:33-52.

17. Mor V, Masterson-Allen S, Goldberg R, et al. Pre-diagnostic symptom recognition and help seeking among cancer patients. J Community Health 1990;15:253-66.

18. Tromp DM, Brouha XD, De Leeuw JR, et al. Psychological factors and patient delay in patients with head and neck cancer. Eur $J$ Cancer 2004;40:1509-16.

19. Lauver D, Tak Y. Optimism and coping with a breast cancer symptom. Nurs Res 1995;44:202-7.

20. de Nooijer J, Lechner L, de Vries H. A qualitative study on detecting cancer symptoms and seeking medical help; an application of Andersen's model of total patient delay. Patient Educ Couns 2001;42:145-57.

21. Rozniatowski O, Reich M, Mallet $Y$, et al. Psychosocial factors involved in delayed consultation by patients with head and neck cancer. Head Neck 2005;27:274-80.

22. Scott SE, Grunfeld EA, Main J, et al. Patient delay in oral cancer: a qualitative study of patients' experiences. Psychooncology 2006;15:474-85. 
23. Scott SE, Walter FM, Webster A, et al. The model of pathways to treatment: conceptualization and integration with existing theory. $\mathrm{Br} \mathrm{J}$ Health Psychol 2013;18:45-65.

24. Walter F, Webster A, Scott S, et al. The Andersen Model of Total Patient Delay: a systematic review of its application in cancer diagnosis. J Health Serv Res Policy 2012; 17:110-18.

25. Becker $\mathrm{MH}$. The health belief model and personal health behavior. Health Educ Monogr 1974;2:324-473.

26. Schwarzer R. Self-efficacy in the adoption and maintenance of health behaviors: theoretical approaches and a new model. In Schwarzer R. ed Self-efficacy: thought control of action. Washington, DC: Hemisphere, 1992:217-43.

27. Leventhal $\mathrm{H}$, Diefenbach $\mathrm{M}$. The active side of illness cognition. In Skelton JACoryle RT. eds Mental representation in health and illness. New York: Springer-Verlag, 1991:247-72.

28. Fishbein M, Ajzen I. Belief, attitude, intention, and behavior: an introduction to theory and research. Reading, MA: Addison-Wesley, 1975.

29. Lazarus RS, Folkman S. Stress, appraisal and coping. New York: Springer, 1984.

30. Noonan B. Understanding the reasons why patients delay seeking treatment for oral cancer symptoms from a primary health care professional: an integrative literature review. Eur $J$ Oncol Nurs 2014;18:118-24.

31. Austoker J, Bankhead C, Forbes LJL, et al. Interventions to promote cancer awareness and early presentation: systematic review. $\mathrm{Br} \mathrm{J}$ Cancer 2009;101:S31-9.

32. Panzarella V, Pizzo G, Calvino F, et al. Diagnostic delay in ora squamous cell carcinoma: the role of cognitive and psychological variables. Int J Oral Sci 2014;6:39-45.
33. Quaife SL, Forbes LJL, Ramirez AJ, et al. Recognition of cancer warning signs and anticipated delay in help-seeking in a population sample of adults in the UK. Br J Cancer 2014;110:12-18.

34. Beeken RJ, Simon AE, von Wagner C, et al. Cancer fatalism: deterring early presentation and increasing social inequalities? Cancer Epidemiol Biomarkers Prev 2011;20:2127-31.

35. Robb K, Stubbings S, Ramirez A, et al. Public awareness of cancer in Britain: a population-based survey of adults. Br J Cancer 2009;101:S18-23.

36. Forbes LJL, Simon AE, Warburton F, et al. Differences in cancer awareness and beliefs between Australia, Canada, Denmark, Norway, Sweden and the UK (the International Cancer Benchmarking Partnership): do they contribute to differences in cancer survival? Br J Cancer 2013;108:292-300.

37. Simon AE, Waller J, Robb K, et al. Patient delay in presentation of possible cancer symptoms: the contribution of knowledge and attitudes in a population sample from the United Kingdom. Cancer Epidemiol Biomarkers Prev 2010;19:2272-7.

38. Scott SE, Grunfeld EA, Auyeung V, et al. Barriers and triggers to seeking help for potentially malignant oral symptoms: implications for interventions. J Public Health Dent 2009;69:34-40.

39. Cox DR. Regression models and life tables. J Royal Stat Soc Series B 1972;34:187-220.

40. Chesher A, Dumangane M, Smith RJ. Duration response measurement error. J Economet 2002;111:1969-94.

41. Abrevaya J, Hausman JA. Semiparametric estimation with mismeasured dependent variables: an application to duration models for unemployment spells. Ann Econ Stat 1999;55/56:243-75.

42. Weller D, Vedsted P, Rubin G, et al. The Aarhus statement: improving design and reporting of studies on early cancer diagnosis. Br J Cancer 2012;106:1262-7. 


\section{BMJ Open}

\section{Determinants of patient delay in doctor consultation in head and neck cancers (Protocol DEREDIA)}

Véronique Christophe, Tanguy Leroy, Mélanie Seillier, et al.

BMJ Open 2014 4:

doi: 10.1136/bmjopen-2014-005286

Updated information and services can be found at:

http://bmjopen.bmj.com/content/4/7/e005286.full.html

These include:

References This article cites 36 articles, 3 of which can be accessed free at: http://bmjopen.bmj.com/content/4/7/e005286.full.html\#ref-list-1

Open Access This is an Open Access article distributed in accordance with the Creative Commons Attribution Non Commercial (CC BY-NC 3.0) license, which permits others to distribute, remix, adapt, build upon this work non-commercially, and license their derivative works on different terms, provided the original work is properly cited and the use is non-commercial. See: http://creativecommons.org/licenses/by-nc/3.0/

Email alerting Receive free email alerts when new articles cite this article. Sign up in service the box at the top right corner of the online article.

Topic Articles on similar topics can be found in the following collections Collections

Notes

To request permissions go to:

http://group.bmj.com/group/rights-licensing/permissions

To order reprints go to:

http://journals.bmj.com/cgi/reprintform

To subscribe to BMJ go to:

http://group.bmj.com/subscribe/ 